\title{
BMJ Open Effectiveness of non-pharmacological interventions to decrease fatigue in people living with HIV/AIDS: a protocol of systematic review and meta- analysis
}

Xueling Xiao (D) , ${ }^{1,2}$ Nancy R Reynolds, ${ }^{2}$ Leorey Saligan, ${ }^{3}$ Yunxiao Lei, ${ }^{4}$ Min Wang, ${ }^{5}$ Honghong Wang ${ }^{1}$

To cite: Xiao X, Reynolds NR, Saligan L, et al. Effectiveness of non-pharmacological interventions to decrease fatigue in people living with HIV/AIDS: a protocol of systematic review and meta-analysis. BMJ Open 2020;10:e040996. doi:10.1136/ bmjopen-2020-040996

- Prepublication history and additional material for this paper is available online. To view these files, please visit the journal online (http://dx.doi.org/10. 1136/bmjopen-2020-040996).

Received 27 May 2020 Revised 22 July 2020 Accepted 13 August 2020
Check for updates

(c) Author(s) (or their employer(s)) 2020. Re-use permitted under CC BY-NC. No commercial re-use. See rights and permissions. Published by BMJ.

For numbered affiliations see end of article.

Correspondence to Professor Min Wang; wangmin2828@163.com

\section{ABSTRACT}

Introduction Fatigue is a common symptom among people living with HIV (PLWH). It has a substantial adverse impact on functional status and the ability to conduct activities of daily living. Identifying effective strategies to prevent or reduce fatigue is significant to promote the quality of life of this vulnerable population. The purpose of this review is to synthesise the non-pharmacological evidence and assess the effects of interventions on reducing HIV-related fatigue among PLWH.

Methods and analysis We will comprehensively search literature available up to 30 June 2020, in the following databases: PubMed, Embase, CINAHL, Cochrane Library, Web of Science and PsycINFO. The reference list of selected studies and relevant published reviews will also be screened to retrieve potential articles. Two reviewers will identify the eligible articles, extract data and identify the biases in the selected studies. Any disagreements will be referred to a third reviewer. We will qualitatively synthesise the evidence and pool data with meta-analysis according to the heterogeneity of different studies.

Ethics and dissemination This systematic review will not raise any ethical issues since it is a secondary data collection and analysis. The results will inform effective strategies to reduce fatigue among PLWH. The final report will be published in a peer-reviewed journal and academic conferences.

PROSPERO registration number CRD42020153715.

\section{BACKGROUND}

Though antiretroviral therapy (ART) has turned HIV into a chronic disease, people living with HIV (PLWH) still suffer from many long-term symptoms. Fatigue is one of the most common, distressing and persistent symptoms that is potentially disabling. It manifests as physical/psychological exhaustion with debilitating effects and causing limitations in one's ability to conduct daily activities. ${ }^{1-3}$ The proportion of PLWH with fatigue is estimated to range from $30 \%$ to as much as $90 \%,{ }^{24}$ which is far more frequent
Strengths and limitations of this study

- To our knowledge, this will be the first review to summarise the non-pharmacological interventions to reduce fatigue among people living with HIV (PLWH) and explore the effectiveness of different strategies.

- The results will provide medical staff, stakeholders and PLWH with a source of high-quality information about options to address fatigue.

- The protocol follows Preferred Reporting Items for Systematic Review and Meta-Analysis Protocols guidelines. Transparent and robust methods will be used to conduct the systematic review and meta-analysis.

Pharmacological interventions will not be included.

than their HIV-negative counterparts. ${ }^{5}$ The presence of fatigue in PLWH contributes to lower quality of life and worsened health outcomes. $^{267}$

Interventions to prevent or treat fatigue among PLWH are being investigated. Use of pharmacological treatments, such as testosterone and psychostimulants, have reduced fatigue among PLWH. ${ }^{8}{ }^{9}$ However, pharmacological interventions are not consistently recommended for HIV-related fatigue, ${ }^{2}$ because the symptom cluster of fatigue is complex $^{38}$; and thus a more multifaceted treatment approach is required. The high prevalence of fatigue in PLWH with corresponding impact on health outcomes prompts an urgent need to develop effective interventions to reduce fatigue in this population.

\section{Description of the condition}

Fatigue in PLWH is persistent and not relieved by rest. ${ }^{10}$ Further, the intensity of fatigue in this population will not remit spontaneously 
according to a 3-year longitudinal study. ${ }^{4}$ The experience of fatigue in PLWH is still not well understood and often not fully acknowledged by health providers. PLWH frequently struggle to implement self-care strategies to manage this distressing symptom. ${ }^{11}$ PLWH with fatigue experience physical weakness that can lead to difficulties self-managing life with HIV. ${ }^{12}$ Fatigue has been associated with less ART adherence and poor health outcomes. ${ }^{13}$

The mechanism of fatigue in PLWH remains elusive. A myriad of physiological, psychological and behavioural factors may contribute to the presence and persistence of fatigue. The relationship between disease progression (eg, CD4 level and viral load) and fatigue in this population had inconsistent results. ${ }^{14-17}$ Other comorbidities, such as cardiovascular disease and metabolic disorders may also contribute to the fatigue experienced by PLWH. ${ }^{18}$ Psychological perspectives, depression, anxiety and stress have also been shown to influence the fatigue experience. ${ }^{81920}$ The overlap of these conditions and behaviours manifests in a cluster with fatigue. ${ }^{3}$ For example, sleep quality has been associated with fatigue in PLWH, ${ }^{21} 22$ particularly, sleep quality moderates the relationship between fatigue patterns and psychological factors, including depression and anxiety. ${ }^{23}$ Prior evidence underscores the complex picture of fatigue in PLWH, which has hindered the development of effective interventions.

\section{Description of intervention}

There is considerable research that has attempted to manage fatigue among people with long-term physical conditions. Previous systematic reviews indicate that exercise and cognitive behavioural therapy (CBT) are common non-pharmacological interventions with potential efficacy in reducing fatigue. ${ }^{24}$ Very few studies have explored fatigue in PLWH, and most of the reviews to date have included multiple physical problems, adding complexity in the interpretation of the results in PLWH. One review focusing on PLWH with advanced illness found that progressive resistive exercise and aerobic exercise were effective but the results cannot be expanded to the general PLWH who are in earlier stages of illness. ${ }^{25}$ A narrative review conducted in 2010 focused on HIVrelated fatigue, but it focused on pharmacological treatment. ${ }^{8}$ In recent trials, various forms of physical exercise ${ }^{26-28}$ and $\mathrm{CBT}^{29}{ }^{30}$ interventions have been examined to explore effect in reducing fatigue in PLWH. One hypothesis that can explain why physical exercise works in reducing fatigue in PLWH is its ability to reserve energy and preserve muscle mass. Similarly, psychological interventions and self-care programmes reduce fatigue in PLWH by managing mood and behaviour. ${ }^{31}$

In summary, fatigue in PLWH is potentially different from that in people with other physical conditions; nevertheless, fatigue induces various limitations. ${ }^{2}$ To our best knowledge, no in-depth systematic review has focused on non-pharmacological interventions specific for HIV-related fatigue. Therefore, this detailed systematic review and meta-analysis will explore evidence of non-pharmacological interventions in reducing fatigue in PLWH and verify the effectiveness of each intervention in reducing HIV-related fatigues.

\section{METHODS AND ANALYSIS \\ Study design}

This protocol is registered with the International Prospective Register of Systematic Reviews. It has complied with the Preferred Reporting Items for Systematic Review and Meta-Analysis Protocols recommendations (PRISMA-P) ${ }^{32}$ The PRISMA-P checklist is shown in the online supplemental file 1 .

\section{Eligibility criteria for selecting studies \\ Types of studies}

This review will include randomised controlled trials (RCTs), quasi-RCTs and controlled before-and-after studies, as defined by the Cochrane Handbook for Systematic Reviews of Interventions. ${ }^{33}$ The randomised and quasi-randomised crossover trials will be included and only results of the first period will be considered. Regarding cluster RCTs, the study with the unit of analysis at an individual level will be included. A full publication in English is required. When multiple studies are based on the same sample, we will include all the papers and staple our interested outcomes together.

\section{Participants}

Participants will be adults (18 years of age or older) with HIV, irrespective of the stage of the HIV infection.

\section{Types of interventions}

We will include trials evaluating non-pharmacological interventions with either the primary or secondary objective to reduce fatigue in PLWH. Studies with interventions combining pharmacological and nonpharmacological components will be included if the data can be extracted separately. We are not limiting related to the setting, provider, timing, frequency and duration of the interventions.

The potential interventions may include, but are not restricted to:

- Cognitive behavioural therapy.

- Self-management strategies.

- Patient education programme.

- Physical training (aerobic training, resistance training, yoga and so on).

\section{Controls}

Trials will be included if they compared an intervention group with controls, which could be placebo, standard care/usual care or wait-list or have a comparison between different interventions with a control.

\section{Outcomes}

The primary outcome of this systematic review will be fatigue of PLWH evaluated as the rate of or the mean 
severity of fatigue. This could be measured by the following tools, but is not limited to:

- HIV-related Fatigue Scale.

- Global Fatigue Index.

- Piper Fatigue Scale.

- Fatigue Severity Scale.

- Lee Fatigue Scale.

If possible, the secondary outcomes will include but will not be restricted to quality of life, psychological health (such as depression and stress), treatment adherence and immune function.

\section{Search strategies}

The literature search will be conducted in the following electronic databases from the inception to 30 June 2020: PubMed, Embase, CINAHL, Cochrane Library, Web of Science and PsycINFO. Our search strategies were developed by XX (the first author) and an academic librarian. The search strategies will include the population of interest (PLWH), the intervention (any non-pharmacological interventions) and the primary outcome of interest (fatigue). The search terms for PubMed were displayed in the online supplemental file 2 to illustrate the logic of the search. Besides the reference list of included studies, relevant published reviews will be explored to retrieve the eligible articles.

\section{Selection of studies}

The identified studies will be imported into COVIDENCE. After removing duplications, two reviewers will independently screen the titles and abstracts, and eliminate records that clearly do not meet the inclusion criteria. The full text of potentially related studies will be obtained and scrutinised for inclusion or exclusion. The excluded studies will be verified by a full text read and be shown in a flow diagram with justifications. Any discrepancies during this process will be solved by the discussion of the two reviewers or by a third arbiter, if necessary. A PRISMA flow will be adopted to detail the information of the screening process. ${ }^{32}$

\section{Data extraction}

A standardised data extraction form has been designed (online supplemental file 3). After piloted and further revised by our team members, this form will be impeded into COVIDENCE (www.covidence.org). The data extraction will be conducted by two reviewers independently. Any inconsistencies will be referred to a third arbiter and discussed to reach a consensus. We will include information about general study information (first author, corresponding author and year of publication), study design (setting, sampling, randomisation, allocation and blinding), sample characteristics (inclusion and exclusion criteria, sample size, age, gender, education background, viral load, $\mathrm{CD} 4^{+}$level, HIV diagnosis duration and drop-off), intervention information (type, content, frequency, duration, provider, control group and timing of follow-ups), and primary and secondary outcomes (measuring time points, tools for assessment, fatigue rate or severity, any abovementioned secondary results). If necessary, we will contact the corresponding author to clarify missing or for more detailed information.

\section{Risk of bias}

Two reviewers will independently assess the risk of bias of the included trials and any disagreements will be discussed to reach a consensus with a third researcher. The methodological quality of the RCTs will be evaluated according to the Revised Cochrane risk-of-bias tool for randomised trials, ${ }^{34}$ which includes five domains, namely risk of bias arising from the randomisation process, risk of bias due to deviation from the intended intervention, risk of bias due to deviations from the intended interventions, missing outcome data, risk of bias in measurement of the outcome, risk of bias in selection of the reported result. The risk of bias of non-RCTs will be assessed based on the Risk of Bias in Non-randomised Studies of Interventions. ${ }^{35}$ This tool includes seven domains: bias due to confounding, bias in selection of participants into study, bias in classification of intervention, bias due to deviation from intended interventions, bias due to missing data, bias in measurement of outcomes and bias in selection of the reported results.

\section{Strategy for data analysis}

We will summarise the main characteristics of the included studies in text and table. A qualitative and narrative synthesis will be implemented to describe how fatigue was defined and measured, and the existing intervention methods, especially the contents, ways of delivery, and efficacy and feasibility of these interventions. If it is possible for a meta-analysis after assessing the extracted data, we will use the Cochrane Review Manager software to conduct statistical analysis. Standardised mean differences and relevant $95 \%$ CI will be calculated for continuous outcomes; risk ratios and relevant $95 \%$ CI will be computed for dichotomous outcomes. If studies reported the severity and rate of fatigue, we will report and calculate both types of outcomes.

According to the controls, we will perform separate analyses for trials: intervention versus standard care and intervention versus blank control. For trials comparing different dosages of intervention with control, we will combine results of the various arms of interventions; otherwise, we will divide the numbers of control group into several parts according to the number of intervention arms to ensure the participants will not be double counted. For trials comparing different types of interventions with control, we will split the control group into several parts as well, one to go with each intervention arm.

The heterogeneity of studies will be checked by $\chi^{2}$ test and $\mathrm{I}^{2}$ test. If the significance of $\chi^{2}$ is smaller than 0.1 or $\mathrm{I}^{2}$ is $\geq 50 \%$, we will use random-effects models to synthesise the results from different articles. Otherwise, we will not need to be concerned with the heterogeneity.

If substantial heterogeneity is present, a qualitative synthesis will be conducted instead.

\section{Subgroup analysis}

We will do subgroup analyses if there are enough trials. It will likely be performed based on characteristics of participants 
(age, gender or depression status), types of intervention (selftraining vs others delivering, or CBT vs exercise training), outcomes relevant features (measurements of fatigues, or severity vs rate of fatigue) and effects of interventions (short term vs long term, or pure non-pharmacological intervention vs non-pharmacological combined with pharmacological interventions). The subgroup analysis will not be limited to those as it highly depends on the extracted data.

\section{Grading quality of evidence}

The Grading of Recommendation Assessment, Development and Evaluation system will guide the evaluation of the evidence of the outcomes. ${ }^{36}$ This system considers the risk of bias, consistency, directness of evidence, precision of effects estimates and publication bias.

\section{Sensitivity analysis}

We plan to explore the sensitivity of the included articles by excluding trials with a high risk of bias.

\section{Assessment of reporting biases}

The funnel plot will be performed to explore the publication biases of the included studies.

\section{Patient and public involvement}

We have no patient and public involvement.

\section{ETHICS AND DISSEMINATION}

We will not use any identifying information of participants in this systematic review to protect the privacy of patients, so no ethical approval is needed. The results will be disseminated by social media (such as WeChat, Twitter, Facebook), academic meetings and publication in peer-reviewed journals.

\section{Author affiliations}

${ }^{1}$ Xiangya Nursing School, Central South University, Changsha, Hunan, China ${ }^{2}$ School of Nursing, Johns Hopkins University, Baltimore, Maryland, USA ${ }^{3}$ National Institute of Nursing Research, National Institutes of Health, Bethesda, Maryland, USA

${ }^{4}$ School of Nursing, Henan University of Science and Technology, Luoyang, Henan, China

${ }^{5}$ HIV/AIDS Department, First Hospital of Changsha, Changsha, Hunan, China

Contributors XX and MW initiated the idea and led the development of the protocol. YL contributed to the methodology development. HW, NRR, MW and LS critically reviewed intellectual content and revised successive drafts of the manuscripts. HW and NRR provided supervision to XX. All the authors approved the publication of the protocol.

Funding This work is financially supported by Innovation-driven project of Central South University (Grant No: 2018zzts244) and China Scholarship Council (Grant No. 201806370164). No other assistance was provided by the funders during the whole process of developing the protocol.

Competing interests None declared.

Patient and public involvement Patients and/or the public were not involved in the design, or conduct, or reporting, or dissemination plans of this research.

Patient consent for publication Not required.

Provenance and peer review Not commissioned; externally peer reviewed.

Open access This is an open access article distributed in accordance with the Creative Commons Attribution Non Commercial (CC BY-NC 4.0) license, which permits others to distribute, remix, adapt, build upon this work non-commercially, and license their derivative works on different terms, provided the original work is properly cited, appropriate credit is given, any changes made indicated, and the use is non-commercial. See: http://creativecommons.org/licenses/by-nc/4.0/.

ORCID iD

Xueling Xiao http://orcid.org/0000-0002-8810-9291

\section{REFERENCES}

1 USNLo M. Fatigue secondary fatigue, 2016. Available: https:// medlineplus.gov/fatigue.html

2 Barroso J, Voss JG. Fatigue in HIV and AIDS: an analysis of evidence. J Assoc Nurses AIDS Care 2013;24:S5-14.

3 Zhu Z, Zhao R, Hu Y. Symptom clusters in people living with HIV: a systematic review. J Pain Symptom Manage 2019;58:115-33.

4 Barroso J, Leserman J, Harmon JL, et al. Fatigue in HIV-infected people: a three-year observational study. J Pain Symptom Manage 2015;50:69-79.

5 Tran T, Guardigni V, Pencina KM, et al. Atypical skeletal muscle profiles in human immunodeficiency virus-infected asymptomatic middle-aged adults. Clin Infect Dis 2018;66:1918-27.

6 Cook PF, Hartson KR, Schmiege SJ, et al. Bidirectional relationships between fatigue and everyday experiences in persons living with HIV. Res Nurs Health 2016;39:154-63.

7 daCosta DiBonaventura M, Gupta S, Cho M, et al. The association of HIV/AIDS treatment side effects with health status, work productivity, and resource use. AIDS Care 2012;24:744-55.

8 Jong E, Oudhoff LA, Epskamp C, et al. Predictors and treatment strategies of HIV-related fatigue in the combined antiretroviral therapy era. AIDS 2010;24:1387-405.

9 Mücke M, Cuhls $\mathrm{H}$, et al, Mochamat. Pharmacological treatments for fatigue associated with palliative care. Cochrane Database Syst Rev 2015:CD006788.

10 Loades ME, Kagee A. Exploring our understanding of fatigue among adolescents living with HIV: highlighting the unknown. $J$ Health Psychol 2019;24:125-36.

11 Jenkin $P$, Koch T, Kralik D. The experience of fatigue for adults living with HIV. J Clin Nurs 2006;15:1123-31.

12 Wang K, Chen W-T, Zhang L, et al. Facilitators of and barriers to HIV self-management: perspectives of HIV-positive women in China. Appl Nurs Res 2016;32:91-7.

13 Al-Dakkak I, Patel S, McCann E, et al. The impact of specific HIV treatment-related adverse events on adherence to antiretroviral therapy: a systematic review and meta-analysis. AIDS Care 2013;25:400-14.

14 Safren SA, Hendriksen ES, Smeaton L, et al. Quality of life among individuals with HIV starting antiretroviral therapy in diverse resourcelimited areas of the world. AIDS Behav 2012;16:266-77.

15 Voss JG. Predictors and correlates of fatigue in HIV/AIDS. J Pain Symptom Manage 2005;29:173-84.

16 Henderson M, Safa F, Easterbrook P, et al. Fatigue among HIVinfected patients in the era of highly active antiretroviral therapy. HIV Med 2005;6:347-52.

17 Lee KA, Portillo CJ, Miramontes H. The fatigue experience for women with human immunodeficiency virus. J Obstet Gynecol Neonatal Nurs 1999;28:193-200.

18 Battegay M, Elzi L. Morbidity and mortality in HIV-infected individuals - a shift towards comorbidities. Swiss Med Wkly 2009;139:564-70.

19 Schifitto G, Deng L, Yeh T-M, et al. Clinical, laboratory, and neuroimaging characteristics of fatigue in HIV-infected individuals. $J$ Neurovirol 2011;17:17-25.

20 Chen W-T, Shiu C-S, Yang JP, et al. Fatigue and sleep disturbance related to perceived stress in Chinese HIV-positive individuals: a mixed methods study. J AIDS Clin Res 2013;4.

21 Salahuddin N, Barroso J, Leserman J, et al. Daytime sleepiness, nighttime sleep quality, stressful life events, and HIV-related fatigue. $J$ Assoc Nurses AIDS Care 2009;20:6-13.

22 Perazzo JD, Webel AR. Barriers to health-promoting behaviour in people living with HIV: implications for comorbidity development. Antiviral therapy 2018;23:A35.

23 Lerdal A, Gay CL, Aouizerat BE, et al. Patterns of morning and evening fatigue among adults with HIV/AIDS. $J$ Clin Nurs 2011;20:2204-16.

24 Hulme K, Safari R, Thomas S, et al. Fatigue interventions in long term, physical health conditions: a scoping review of systematic reviews. PLoS One 2018;13:e0203367.

25 Payne C, Wiffen PJ, Martin S. Withdrawn: interventions for fatigue and weight loss in adults with advanced progressive illness. Cochrane Database Syst Rev 2017;4:CD008427. 
26 Nosrat S, Whitworth JW, Dunsiger SI, et al. Acute effects of resistance exercise in a depressed HIV sample: the exercise for people who are immunocompromised (EPIC) study. Ment Health Phys Act 2017:12:2-9.

27 Nosrat S. Acute effects of resistance exercise intensity in a depressed HIV sample: the exercise for people who are immunocompromised (EPIC) study. ProQuest Information \& Learning 2018.

28 Jaggers JR, Hand GA, Dudgeon WD, et al. Aerobic and resistance training improves mood state among adults living with HIV. Int $J$ Sports Med 2015;36:175-81.

29 Doerfler RE, Goodfellow L. Brief exposure to cognitive behavioral therapy reduces side-effect symptoms in patients on antiretroviral therapy. J Assoc Nurses AIDS Care 2016;27:455-67.

30 Doerfler RE. Clinical trial of cognitive-behavioral therapy to reduce antiretroviral effects in HIV patients. ProQuest Information \& Learning 2011.

31 Marie Modeste RR, Majeke SJ. Self-Care symptom-management strategies amongst women living with HIV /AIDS in an urban area in KwaZulu-Natal. Health SA Gesondheid 2010;15:162-9.
32 Moher D, Shamseer L, Clarke M, et al. Preferred reporting items for systematic review and meta-analysis protocols (PRISMA-P) 2015 statement. Syst Rev 2015;4:1.

33 Cumpston M, Li T, Page MJ, et al. Updated guidance for trusted systematic reviews: a new edition of the Cochrane Handbook for systematic reviews of interventions. Cochrane Database Syst Rev 2019;10:ED000142.

34 Higgins JPT, Savović J, Page MJ, et al. Revised Cochrane risk-ofbias tool for randomized trials (RoB 2). Secondary revised Cochrane risk-of-bias tool for randomized trials (RoB 2), 2019. Available: https://sites.google.com/site/riskofbiastool/welcome/rob-2-0-tool/ current-version-of-rob-2

35 Sterne JA, Hernán MA, Reeves BC, et al. ROBINS-I: a tool for assessing risk of bias in non-randomised studies of interventions. BMJ 2016;355:i4919.

36 Guyatt GH, Oxman AD, Vist GE, et al. GRADE: an emerging consensus on rating quality of evidence and strength of recommendations. BMJ 2008;336:924-6. 